are greatly suppressed (curves ii and iii in Fig. 3a). Both channels exhibit small leakage. We attribute the first peak to photons retrieved near the cell boundaries, which do not experience sufficient Bragg reflections to be trapped efficiently. The long tail is probably due to a slow spreading of the stored pulse. When the backward beam is turned off, the released pulse is detected in the forward channel (curve ii). The presence of signal light inside the cell during the simultaneous application of the two control beams was verified directly by monitoring fluorescence from the side of the cell (Fig. 3b). For times when the signal output in the forward and backward directions is greatly suppressed, we observed significant enhancement of the signal light fluorescence (curve iii in Fig. 3b), due to residual atomic absorption.

These observations provide evidence for controlled conversion of the stored atomic coherence into a stationary photonic excitation in the cell. Note, in particular, that the magnitude of the fluorescence drops sharply after the backward pulse is turned off. This drop is followed by a gradual decay, associated with the exit of the slow pulse from the medium. This behaviour is in qualitative agreement with our simple model, which predicts the light intensity in the stationary pulses to be double that in the slowly propagating pulse. As shown in Fig. 3c, the magnitude of the released pulse decreases exponentially with increasing trapping time with a characteristic time constant of about $7 \mu \mathrm{s}$. Note that only a part of this decay is due to the hyperfine coherence decay. Other decay mechanisms include spreading of the stationary pulse, as well as imperfect EIT. We anticipate that improvements in efficiency can probably be achieved by initial optical pumping into a single atomic sublevel, using an atomic system with larger level spacing or using the sharper absorption lines of cold atom clouds.

We finally outline possible avenues opened by the present work. First, we note that our procedure is based on a passive medium, and in the ideal limit is not accompanied by optical loss or gain, hence avoiding the associated noise. We therefore anticipate that our method preserves the quantum states of light pulses. (This is in contrast to Bragg gratings based on gain modulation ${ }^{21}$.) Second, although the present work demonstrates stationary light localization and storage in one dimension, it should be possible to controllably localize and guide stationary photonic pulses in three spatial dimensions by using control beams with properly designed wavefronts. Third, controlled conversion of propagating light into stationary light pulses opens interesting possibilities for enhanced nonlinear optical processes by combining the present technique with the resonant enhancement of nonlinear optics via EIT ${ }^{24-26}$. This combination may enable controlled interactions involving quantum few-photon field $\mathrm{s}^{27-30}$ analogous to those feasible in cavity quantum electrodynamics ${ }^{5}$. Finally, extension of the present ideas to other systems might be possible using, for example, dynamic modulation of photonic bandgap materials.

Received 6 September; accepted 4 November 2003; doi:10.1038/nature02176

1. Scully, M. O. \& Zubairy, S. M. Quantum Optics (Cambridge Univ. Press, Cambridge, UK, 1997).

2. Harris, S. E. Electromagnetically induced transparency. Phys. Today 50(7), 36-42 (1997)

3. Lukin, M. D. \& Imamoglu, A. Controlling photons using electromagnetically induced transparency. Nature 413, 273-276 (2001).

4. Matsko, A. B. et al. Slow, ultraslow, stored, and frozen light. Adv. At. Mol. Opt. Phys. 46, 191-242 (2001)

5. Mabuchi, H. \& Doherty, A. C. Cavity quantum electrodynamics: Coherence in context. Science 298, 1372-1377 (2002)

6. Cirac, J. I. et al. Quantum state transfer and entaglement distribution among distant nodes in a quantum network. Phys. Rev. Lett. 78, 3221-3224 (1997).

7. Kuzmich, A. et al. Generation of nonclassical photon pairs for scalable quantum communication with atomic ensembles. Nature 423, 731-734 (2003).

8. van der Wal, C. H. et al. Atomic memory for correlated photon states. Science 301, 196-200 (2003).

9. Boyd, R. W. Nonlinear Optics (Academic, New York, 1992).

10. Boller, K. J., Imamoglu, A. \& Harris, S. E. Observation of electromagnetically induced transparency. Phys. Rev. Lett. 66, 2593-2596 (1991).

11. Hau, L. V. et al. Light speed reduction to 17 metres per second in an ultracold atomic gas. Nature 397, 594-598 (1999).

12. Kash, M. M. et al. Ultraslow group velocity and enhanced nonlinear optical effects in a coherently driven hot atomic gas. Phys. Rev. Lett. 82, 5229-5232 (1999).

13. Fleischhauer, M. \& Lukin, M. D. Dark-state polaritons in electromagnetically induced transparency.
Phys. Rev. Lett. 84, 5094-5097 (2000).

14. Liu, C. et al. Observation of coherent optical information storage in an atomic medium using halted light pulses. Nature 409, 490-493 (2001)

15. Phillips, D. F. et al. Storage of light in atomic vapor. Phys. Rev. Lett. 86, $783-786$ (2001).

16. Zibrov, A. S. et al. Transporting and time reversing light via atomic coherence. Phys. Rev. Lett. 88, 103601 (2002).

17. Kocharovskaya, O., Rostovtsev, Y. \& Scully, M. O. Stopping light via hot atoms. Phys. Rev. Lett. 86, 628-631 (2001).

18. Andre, A. \& Lukin, M. D. Manipulating light pulses via dynamically controlled photonic band gas. Phys. Rev. Lett. 89, 143602 (2002).

19. Harris, S. E. Electromagnetically induced transparency with matched pulses. Phys. Rev. Lett. 70, 552-555 (1993).

20. Cao, H. et al. Random laser action in semiconductor powder. Phys. Rev. Lett. 82, 2278-2281 (1999).

21. Kogelnik, H. \& Shank, C. V. Coupled wave theory of distributed feedback lasers. J. Appl. Phys. 43, 2327-2335 (1972).

22. Yablonovich, E. et al. Donor and acceptor modes in photonic bandgap structures. Phys. Rev. Lett. 67, 3380-3383 (1991).

23. Slusher, D. \& Eggelton, B. (eds) Nonlinear Photonic Crystals (Springer, New York, 2003).

24. Harris, S. E., Field, J. E. \& Imamoglu, A. Nonlinear optical processes using electromagnetically induced transparency. Phys. Rev. Lett. 64, 1107-1110 (1990).

25. Schmidt, H. \& Imamoglu, A. Giant Kerr nonlinearity obtained by electromagnetically induced transparency. Opt Lett. 21, 1936-1938 (1996).

26. Hemmer, P. R. et al. Efficient low-intensity optical-phase conjugation based on coherent population trapping in sodium. Opt. Lett. 20, 982-984 (1995).

27. Harris, S. E. \& Hau, L. V. Nonlinear optics at low light levels. Phys. Rev. Lett. 82, $4611-4614$ (1999). 28. Lukin, M. D. \& Imamoglu, A. Nonlinear optics and quantum entanglement of ultraslow single photons. Phys. Rev. Lett. 84, 1419-1422 (2000).

29. Petrosyan, D. \& Kurizki, G. Symmetric photon-photon coupling by atoms with Zeeman-split sublevels. Phys. Rev. A 65, 033833 (2002).

30. Ottaviani, C., Vitali, D., Artoni, M., Cataliotti, F. \& Tombesi, P. Polarization qubit phase gate in driven atomic media. Phys. Rev. Lett. 90, 197902 (2003).

Acknowledgements We thank A. Andre, M. Eisaman, L. Childress, C. van der Wal, R. Walsworth, S. Zibrov and T. Zibrova for discussions, experimental help and comments on the manuscript. This work is supported by the NSF, the DARPA, the David and Lucille Packard Foundation and the Alfred Sloan Foundation. Partial support by the ONR (DURIP) is also acknowledged.

Competing interests statement The authors declare that they have no competing financial interests.

Correspondence and requests for materials should be addressed to M.D.L. (lukin@physics.harvard.edu).

\section{An off-normal fibre-like texture in thin films on single-crystal substrates}

\section{Detavernier ${ }^{1,2}$, A. S. Özcan ${ }^{3}$, J. Jordan-Sweet ${ }^{1}$, E. A. Stach ${ }^{4}$, J. Tersoff ${ }^{1}$, F. M. Ross $^{1} \&$ C. Lavoie ${ }^{1}$}

${ }^{1}$ IBM T. J. Watson Research Center, Yorktown Heights, New York, New York 10598, USA

${ }^{2}$ Department of Solid State Physics, Ghent University, 9000 Ghent, Belgium ${ }^{3}$ Department of Physics, Boston University, Boston, Massachusetts 02215, USA ${ }^{4}$ National Center for Electron Microscopy, Lawrence Berkeley National Laboratory, Berkeley, California 94720, USA

In the context of materials science, texture describes the statistical distribution of grain orientations. It is an important characteristic of the microstructure of polycrystalline films ${ }^{1-5}$, determining various electrical, magnetic and mechanical properties. Three types of texture component are usually distinguished in thin films: random texture, when grains have no preferred orientation; fibre texture ${ }^{6-10}$, for which one crystallographic axis of the film is parallel to the substrate normal, while there is a rotational degree of freedom around the fibre axis; and epitaxial alignment (or in-plane texture) on single-crystal substrates ${ }^{11-15}$, where an in-plane alignment fixes all three axes of the grain with respect to the substrate. Here we report a fourth type of texture-which we call axiotaxy-identified from complex but 
symmetrical patterns of lines on diffraction pole figures for thin films formed by solid-state reactions. The texture is characterized by the alignment of planes in the film and substrate that share the same $d$-spacing. This preferred alignment of planes across the interface manifests itself as a fibre texture lying off-normal to the sample surface, with the fibre axis perpendicular to certain planes in the substrate. This texture forms because it results in an interface, which is periodic in one dimension, preserved independently of interfacial curvature. This new type of preferred orientation may be the dominant type of texture for a wide class of materials and crystal structures.

Texture is frequently studied using X-ray diffraction (XRD). A pole figure for a particular $(h k l)$ plane depicts the statistical angular distribution of the direction of the normal to this plane ${ }^{1-3}$. One can imagine placing the sample in the centre of an imaginary hemisphere and, for each grain in the film, marking the intersection between the normal to the chosen $(h k l)$ plane and the sphere. By projecting the density of marks on the sphere onto a planar surface, one obtains the ( $h k l)$ pole figure (Fig. la). The angles $\phi$ and $\chi$ are defined as spherical coordinates (azimuth and elevation) in the hemisphere.

Pole figures for thin films typically consist of simple geometric features (Fig. 1b, c). Random texture results in featureless pole figures. Fibre texture ${ }^{4-10}$ (Fig. 1b) can be identified by the occurrence of circles centred around the middle of the pole figure. (For some materials, the fibre axis is at a small angle to the surface normal ${ }^{16-18}$; this variant of fibre texture is sometimes referred to as tilted texture.) Epitaxial alignment (also referred to as 'in-plane alignment' or 'in-plane texture') ${ }^{11-15}$ can be identified by the presence of well-defined spots at certain locations on the pole figure (Fig. 1c). The three types of texture (random, fibre texture and epitaxial) are frequently observed simultaneously within the same film. For instance, multiple variants of in-plane alignment can coexist within a $\mathrm{CoSi}_{2}$ film on $\mathrm{Si}(100)$ (refs 11, 12). When different texture
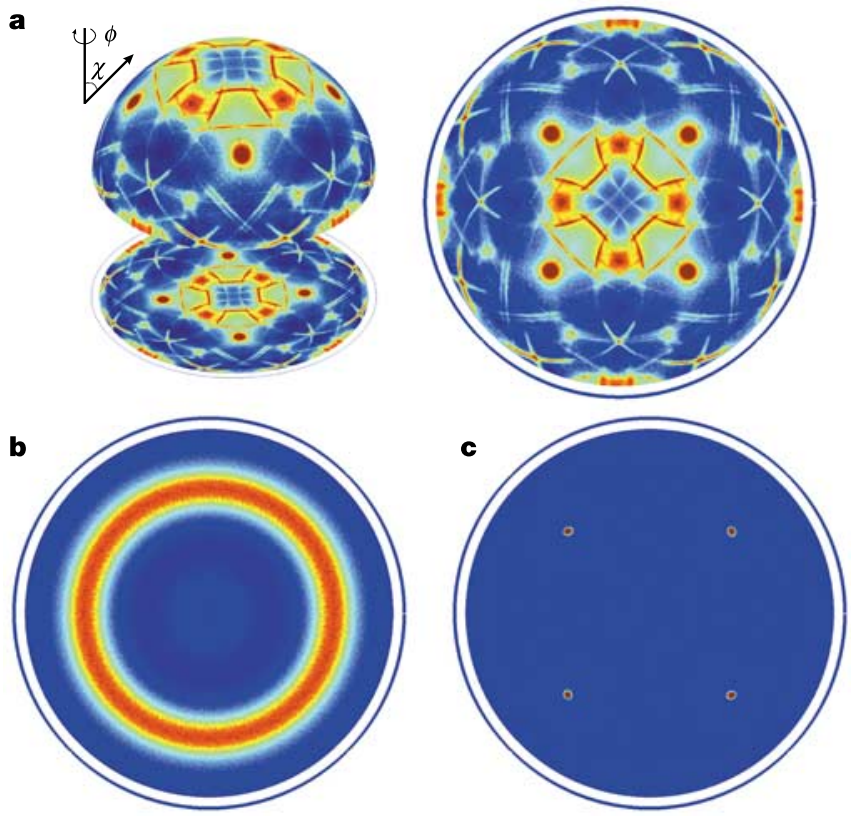

Figure 1 Comparison with standard thin-film pole figures. a, Illustration of the projection method (left) used to obtain the NiSi(112) pole figure (right) for a 60-nm NiSi layer formed by annealing in ultrahigh vacuum. b. Example of 'standard' fibre texture: a Cu(200) pole figure illustrating (111)-fibre texture for a 500-nm-thick film of Cu sputter deposited on $\mathrm{SiO}_{2}$ c, Example of 'standard' epitaxial alignment: a $\mathrm{Cu}(111)$ pole figure showing the epitaxial alignment of a 500-nm Cu film deposited on HF-cleaned Si(001). Regions of high (low) diffracted intensity are coloured red (blue). components are present within the same film, each of these texture components will contribute to the pole figures, which will then consist of different sets of rings and/or spots.

Here we report on intriguingly complex pole figures containing symmetrical line patterns that were measured for several materials formed by a solid-state reaction on a single-crystal substrate. These lines, evident in the spherical representation of the $\mathrm{NiSi}(112)$ pole figure in Fig. 1a, are shown in more detail in Fig. 2a-c, which illustrates three pole figures for a $60-\mathrm{nm} \mathrm{NiSi}$ film on $\mathrm{Si}(001)$. The figures represent the raw data, without any smoothing, fitting, noise reduction or other data enhancement techniques. The patterns of lines cannot be described by either fibre texture (which would result in concentric circles), epitaxial alignment (which would result in spots) or by any combination of a discrete set of known types of texture. Therefore, these patterns are characteristic of a new type of texture, for which we propose the name axiotaxy. Although we only show results here for a 60 -nm-thick film of orthorhombic NiSi on $\mathrm{Si}(001)$, the occurrence of axiotaxy appears to be a robust phenomenon. It is determined primarily by the selection of film and
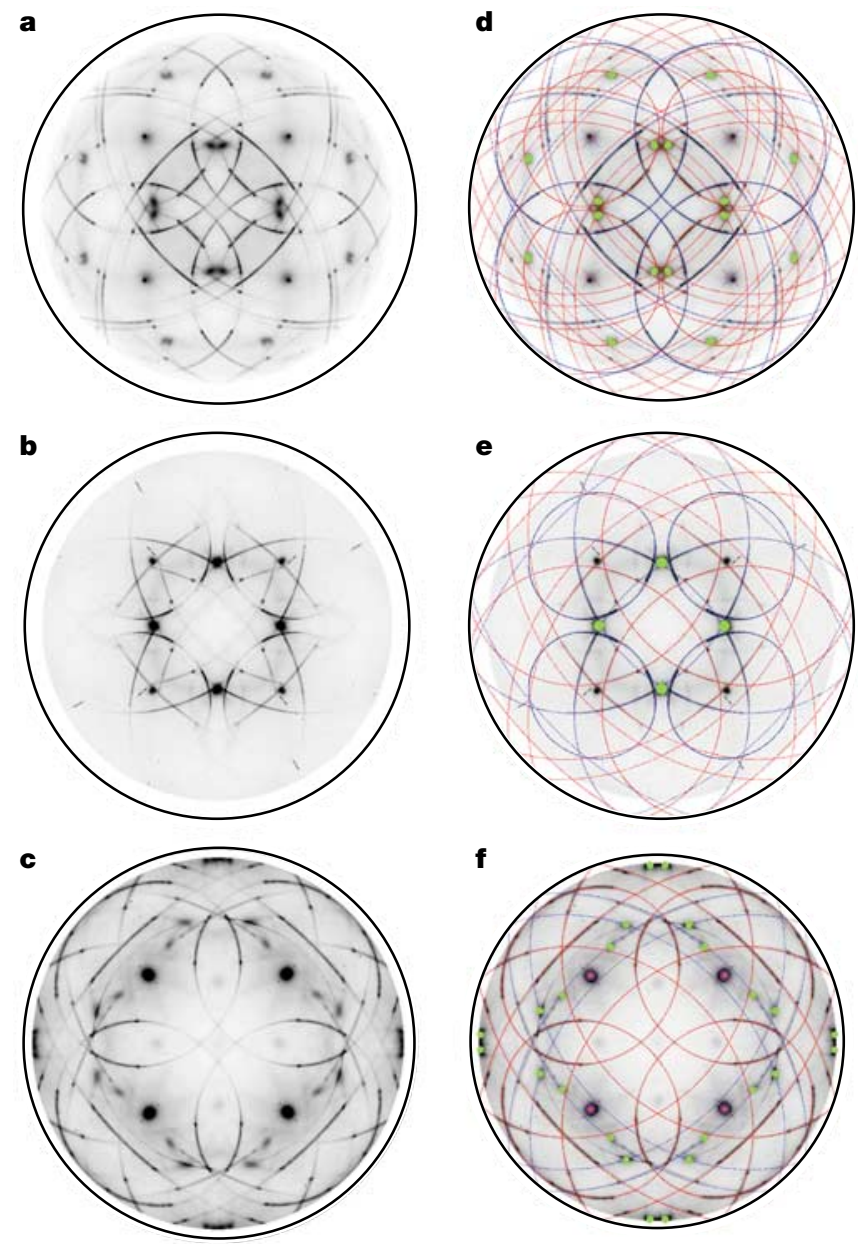

Figure 2 Pole figures and calculated pattern of lines for a NiSi film on Si(001). a-c, Pole figures for a 60-nm NiSi film on Si(001); a, (112); b, (103); and c, (202)/(211). The film was formed by annealing in nitrogen for $30 \mathrm{~s}$ at $550^{\circ} \mathrm{C}$. The diffracted intensity is shown on a linear grey-scale ranging from 0 (white) to 3,000 (black) counts above the background. $\mathbf{d}-\mathbf{f}$, The same data with calculated line patterns overlaid on top of the measurement. These patterns were calculated assuming that NiSi(211) (red lines) or (202) planes (blue lines) are parallel to $\mathrm{Si}(110)$-type planes in the substrate (that is, fibre axes at $\chi=45^{\circ} ; \phi=45^{\circ}, 135^{\circ}, 225^{\circ}, 315^{\circ}$ ). Green dots, the calculated pattern for the epitaxial texture component (see text); magenta crosses, location of signal from Si substrate peaks. 
substrate material, and to first order is independent of processing conditions. Indeed, we observed similar features for NiSi films on $\mathrm{Si}(001),(111)$ or (110) substrates with film thickness ranging from 16 to $200 \mathrm{~nm}$, annealed in nitrogen, purified helium or ultrahigh vacuum at temperatures ranging from 400 to $750{ }^{\circ} \mathrm{C}$. This indicates that the observations reported here are not related to specific annealing conditions or the presence of impurities. The solidstate reaction between the deposited Ni film and the Si substrate is known to result in a phase sequence, with $\mathrm{Ni}$ first transforming into metal-rich silicides, followed by transformation into $\mathrm{NiSi}$ at higher temperature by continued Ni diffusion. It could be thought that the observed texture of $\mathrm{NiSi}$ is related to this specific formation sequence, owing to (for example) some particular property of the precursor phase. However, we observed similarly complex patterns on pole figures for other films also formed by solid-state reaction (tetragonal $\alpha-\mathrm{FeSi}_{2} / \mathrm{Si}(001)$, cubic $\mathrm{CoSi}_{2} / \mathrm{Si}(001)$, orthorhombic $\mathrm{NiGe} / \mathrm{Ge}(001)$ and orthorhombic $\left.\mathrm{C} 54-\mathrm{TiSi}_{2} / \mathrm{Si}(001)\right)$, indicating that the phenomenon of axiotaxy is not related to some peculiarity of a single material (for example, the texture of a specific precursor phase, or a diffusion- versus nucleation-controlled formation process) or even of a single class of crystal structures (Supplementary Figs A1 and A2).

The pole figures (Fig. 2) allow unambiguous identification of the preferential orientation of the grains with respect to the reference frame provided by the single-crystal substrate. The features appear circular on the spherical representation in Fig. 1a, so this suggests that the patterns of lines are related to a fibre-like texture with the fibre axes at $\chi=45^{\circ}$ (instead of normal to the substrate-that is, at $\chi=0^{\circ}$-as for standard fibre texture). Indeed, we found that the complex patterns on Figs 1a and 2 are generated by two off-normal fibre textures, each with fibre axes at $\chi=45^{\circ}$ and four symmetryrelated $\phi$ values $\left(45^{\circ}, 135^{\circ}, 225^{\circ}, 315^{\circ}\right)$. As the fibre axes are aligned along $\mathrm{Si}(110)$-type directions in the substrate, the preferential orientation of the grains that are part of the axiotaxy components

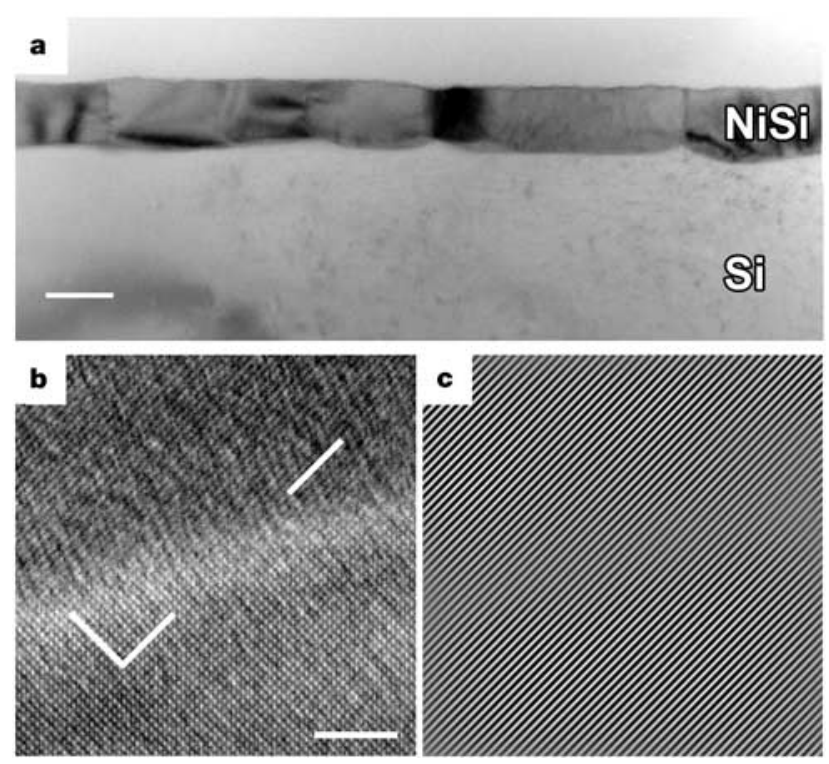

Figure 3 Transmission electron micrographs of axiotaxial NiSi grains. a, Lowmagnification image showing the grain structure. The typical grain size is $100 \mathrm{~nm}$, and some grain boundary grooving is visible. Scale bar, $50 \mathrm{~nm}$. b. Higher-resolution image showing the correspondence of lattice planes in the Si and NiSi. The image has been Wiener filtered to slightly enhance lattice fringe contrast ${ }^{20}$. The orientations of the NiSi and Si are shown, and the alignment of the lattice planes across the interface is indicated. Scale bar, $2.5 \mathrm{~nm}$. c. A filtered version of $\mathbf{b}$ where all intensity is removed from the image apart from that associated with the NiSi(211) / (202) and Si(110) periodicity. This filtering allows us to confirm visually the alignment of these planes. is defined by the constraint of aligning NiSi (202) or (211) planes in the film parallel to $\mathrm{Si}(110)$-type planes in the substrate.

The intricate geometrical patterns in the data (Fig. 2a-c) are reproduced in Fig. $2 \mathrm{~d}-\mathrm{f}$ with calculated patterns for the three main texture components overlaid on the measured patterns, illustrating the excellent correspondence. The calculations should not be considered as curve fitting because they are based purely on geometry, without any adjustable parameters. In addition to the two axiotaxy components, the pole figures contain intense spots that are not part of the lines. These spots are caused by a standard epitaxial alignment, for which the $\mathrm{NiSi}(200)$ plane is almost parallel to $\mathrm{Si}(010)$ and the $\mathrm{NiSi}(014)$ plane is parallel to $\mathrm{Si}(001)$. A transmission electron microscopy (TEM) cross-section for such a grain is shown in Supplementary Fig. A3.

We characterized the morphology of the NiSi grains using crosssectional TEM analysis. The film consists of a single layer of grains typically $100 \mathrm{~nm}$ across (Fig. 3a). The interface between the NiSi and the substrate is abrupt (that is, no amorphous interlayer) and not faceted. Considerable grain boundary grooving was observed, indicating that for this particular annealing temperature, agglomeration had already started, and the NiSi/Si interface was therefore not flat. The high-resolution TEM micrograph of Fig. 3b provides direct corroborating evidence for the preferred alignment of $\mathrm{NiSi}(202)$ or (211) planes parallel to Si(220) planes. To show this more clearly, the image was filtered to keep only intensity associated with the $\mathrm{NiSi}(211) /(202)$ and $\mathrm{Si}(110)$ periodicity (Fig. 3c), confirming the alignment of the planes in the film and substrate.

Although an understanding of the crystallographic orientation of

a

$\begin{array}{rllr}\operatorname{NiSi}(103) & \mathrm{NiSi}(211) & \mathrm{NiSi}(202) & \mathrm{NiSi}(112) \\ d=1.78 \AA & d=1.92 \AA & d=1.92 \AA & d=1.98 \AA \\ d_{\mathrm{p}}=2.72 \AA & d_{\mathrm{p}}=2.71 \AA & d_{\mathrm{p}}=2.72 \AA & d_{\mathrm{p}}=2.73 \AA\end{array}$

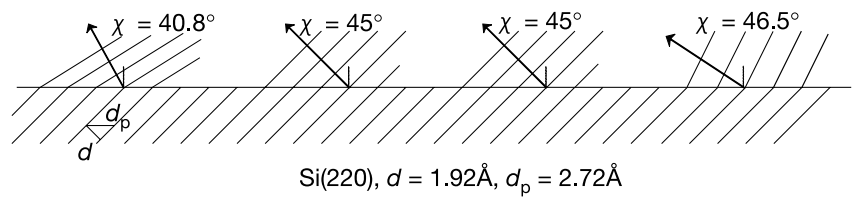

b

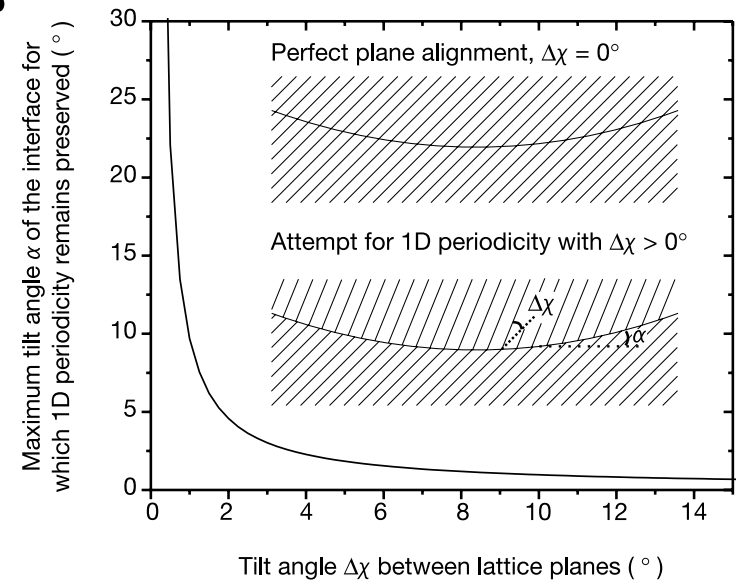

Figure 4 Illustration of the importance of plane alignment across the interface. a, A 1D periodic interface can be achieved by compensating the mismatch in $d$-spacing by tilting the lattice planes over an angle $\Delta \chi$, thus reducing the mismatch in projected $d$-spacing, $\Delta d_{\mathrm{p}}$. However, this works only for a flat interface (insets in $\mathbf{b}$ ). $\mathbf{b}$, The graph shows the maximum angle $\alpha$ through which the interface can curve while maintaining good $1 \mathrm{D}$ periodicity (defined as mismatch $\Delta d_{\mathrm{p}}<0.5 \%$ ), as a function of the tilt angle $\Delta \chi$. The larger the allowed value of $\alpha$, the more stable the $1 \mathrm{D}$ periodic nature of the interface with respect to interfacial curvature. 
the textured grains is interesting in itself, one is left to wonder why plane alignment across the interface is preferred, and why specifically the $\mathrm{NiSi}(202)$ and (211) planes are selected. We note that the interplanar spacings of 1.921 and $1.919 \AA$ for $\mathrm{NiSi}(202)$ and $(211)$ planes, respectively ${ }^{19}$, are within $0.06 \%$ of the spacing of $1.9201 \mathrm{~A}$ for $\mathrm{Si}(220)$ planes, and the alignment of planes with almost identical $d$-spacing across the interface clearly results in a structure that is periodic along one direction in the plane of the interface (Fig. 3b).

However, unlike fibre texture and epitaxy, for which a single $(h k l)$ plane is preferentially parallel to the interface, the interfaces of grains belonging to the same axiotaxy component consist of different crystallographic planes, depending on the rotation around the off-normal fibre axis. Any such rotation produces an interface that remains periodic along at least one direction, but some rotations will result in a higher degree of interfacial periodicity. In fact, we observe that the diffracted intensity varies along the arcs on the pole figures. We calculate that the most intense spots on the lines are caused by grain orientations for which periodicity is achieved along two independent directions in the plane of the interface (Supplementary Fig. A4).

Axiotaxy, resulting in a one-dimensional (1D) periodic interface with a possibly lower-energy bond configuration, can thus be considered as an intermediate case between a random interface (no periodicity) and epitaxy (a two-dimensional, 2D, periodic interface). However, it is important to note that although 1D periodicity can in principle be achieved for any combination of substrate and film planes by choosing an appropriate tilt angle $\Delta \chi$ (Fig. 4a shows some options for different NiSi planes), axiotaxy is experimentally observed only for $1 \mathrm{D}$ periodic interface structures that result from plane alignment across the interface (that is, with $\Delta \chi \approx 0^{\circ}$ ). To explain the apparent importance of plane alignment, we consider the effect of interfacial roughness (Fig. $4 \mathrm{~b}$ insets). The interface of thin films is rarely perfectly flat, especially for films formed by a solid-state reaction. Suppose that at one point on a curved grain there is good $1 \mathrm{D}$ periodicity $(0 \%$ mismatch) because of a properly chosen tilt angle $\Delta \chi$. Because of interface curvature, the projected $d$-spacing changes as one moves away from this point, reducing the quality of the $1 \mathrm{D}$ periodic matching (lower inset). One can calculate the mismatch in projected $d$-spacing $\Delta d_{\mathrm{p}}$ along the interface as a function of the tilt angle $\Delta \chi$ and an angle $\alpha$ that reflects the curvature of the interface (Fig. $4 \mathrm{~b}$, and Supplementary Fig. A5). For perfect plane alignment across the interface, $\Delta \chi=0^{\circ}$ and $\alpha$ can be arbitrarily large, indicating that the $1 \mathrm{D}$ periodic nature of the interface remains preserved irrespective of interfacial roughness (upper inset). However, if $\Delta \chi$ is not zero, then as the interface curves away from a point with zero mismatch, the mismatch increases. If we select a mismatch of $<0.5 \%$ as our criterion for good periodicity, Fig. $4 \mathrm{~b}$ shows that for increasing $\Delta \chi$, the range of curvature over which the interface maintains good periodicity shrinks dramatically. We may conclude that (nearly) perfect alignment of planes in film and substrate that share (nearly) the same $d$-spacing is the only way in which a $1 \mathrm{D}$ periodic interface structure can be achieved, the periodic nature of which is preserved independently of interfacial curvature.

To corroborate the above model, we consider two weak axiotaxyrelated texture components that are observed in the pole figures for the NiSi film when plotting the data using a logarithmic intensity scale (Supplementary Figs A6 and A7). These two additional components are caused by off-normal fibre texture related to $\mathrm{NiSi}(103)$ and (112) planes for which the fibre axis is at $\chi=40.8^{\circ}$ and $\chi=46.5^{\circ}$, respectively, and $\phi=45^{\circ}, 135^{\circ}, 225^{\circ}, 315^{\circ}$. NiSi $(103)$ has a $d$-spacing $7.6 \%$ smaller than $\operatorname{Si}(220)$, while $\mathrm{NiSi}(112)$ has a $d$-spacing $3 \%$ larger than $\mathrm{Si}(220)$, and this mismatch has been compensated by slightly tilting the NiSi planes (Fig. 4a). Indeed, the measured tilts of $\Delta \chi=-4.2^{\circ}$ for $\mathrm{NiSi}(103)$ and $\Delta \chi=+1.5^{\circ}$ for $\mathrm{NiSi}(112)$ reduce the mismatch $\Delta d_{\mathrm{p}}$ in $d$-spacing projected onto the interface to only $-0.01 \%$ and $0.4 \%$, respectively. This illustrates the importance of achieving a 1D periodic interface structure (with $\left.\Delta d_{\mathrm{p}} \approx 0 \%\right)$ in providing the driving force for this type of offnormal fibre texture. However, the relatively weak and broad intensities of these two axiotaxy components, compared with the $\mathrm{NiSi}(202)$ or (211) components, corroborate the importance of plane alignment, as explained by the model.

We finally comment that the occurrence of axiotaxy may be controlled by adjusting the interplanar spacing of lattice planes (Supplementary Fig. A8). Adding Pt to the $\mathrm{Ni}-\mathrm{Si}$ reaction is known to result in the formation of a solid solution with a NiSi-type structure with a larger unit cell than pure NiSi. Alloying with 5-10\% Pt causes the (112)-related axiotaxy to disappear (as the increase in $d$-spacing would require an even higher tilt angle) and the (202)/ (211)-related axiotaxy to weaken, while the (103) axiotaxy component becomes more defined and stronger in intensity as the (103) plane spacing increases and the planes become more parallel to $\operatorname{Si}(220)$ (that is, $\Delta \chi$ decreases).

\section{Methods}

\section{Sample preparation}

Films of $8-100 \mathrm{~nm}$ of $\mathrm{Ni}, 30 \mathrm{~nm}$ of $\mathrm{Ni}(5 \% \mathrm{Pt}), \mathrm{Fe}$, Co and $\mathrm{Co}(5 \% \mathrm{Ti})$ were sputter deposited onto $\mathrm{HF}$-cleaned $\mathrm{Si}(001)$ substrates in an ultrahigh-vacuum deposition system. The samples were annealed in nitrogen ambient for $30 \mathrm{~s}$ at $950{ }^{\circ} \mathrm{C}$ (for Fe and Co) or $550^{\circ} \mathrm{C}$ (for Ni). In addition, a 60-nm-thick NiSi film was formed by annealing in situ-that is, in ultrahigh vacuum (pressure $7 \times 10^{-10}$ torr at the start of the anneal, heater held at $550{ }^{\circ} \mathrm{C}$ for $5 \mathrm{~min}$, pressure increased to $1.6 \times 10^{-9}$ torr at the end of the anneal). Standard $\theta / 2 \theta$ $\mathrm{X}$-ray diffraction was used to confirm that annealing resulted in the formation of $\alpha-\mathrm{FeSi}_{2}$, $\mathrm{CoSi}_{2}$ or NiSi, respectively, with no evidence of other phases present in the films.

\section{Experimental procedure}

Pole figures were measured at the X20A beamline of the National Synchrotron Light Source at Brookhaven National Laboratory. A Si monochromator was used to select a wavelength of $1.5406 \AA$. This wavelength was chosen to simplify comparison with laboratory-based measurements using $\mathrm{CuK} \alpha$ radiation. The sample was mounted on a four-circle diffractometer (Schultz geometry). We used a scintillation counter to detect the diffracted intensity. By fixing the sample and detector at a given $\theta$ and $2 \theta$ angle, one fixes the $d$-spacing of the crystallographic plane in the film for which diffraction will be detected. The pole figure is then obtained by rotating the sample around the axis that is normal to its surface ( $\phi$ scan) and around the axis that is formed by the intersection of the sample surface and the plane defined by the X-ray beam and detector ( $\chi$ scan). The pole figures were acquired in steps of $0.5^{\circ}$ in $\phi$ and $\chi\left(0^{\circ} \leq \phi \leq 90^{\circ}\right.$ and $\left.0 \leq \chi \leq 85^{\circ}\right)$. $\phi$ and $\chi$ alignment was achieved by setting $\phi$ and $\chi$ equal to $45^{\circ}$ at the location of the $\operatorname{Si}(011)$ substrate peak. As a consequence, the directions $\left(\phi=0^{\circ}, \chi=0^{\circ}\right),\left(\phi=0^{\circ}, \chi=90^{\circ}\right)$ and $\left(\phi=90^{\circ}, \chi=90^{\circ}\right)$ correspond to the directions of the normal to the $\operatorname{Si}(001),(\overline{1} 10)$ and (110) planes, respectively. Transmission electron microscopy was carried out at $800 \mathrm{kV}$ accelerating voltage on the Atomic Resolution Microscope (which has a point resolution of $0.16 \mathrm{~nm}$ ) at the National Center for Electron Microscopy, Lawrence Berkeley National Laboratory.

Received 20 March; accepted 4 November 2003; doi:10.1038/nature02198.

1. Kocks, U. F., Tome, C. N. \& Wenk, H.-R. Texture and Anisotropy: Preferred Orientations in Polycrystals and their Effect on Materials Properties (Cambridge Univ. Press, Cambridge, UK, 1976).

2. Randle, V. \& Engler, O. Introduction to Texture Analysis: Macrotexture, Microtexture and Orientation Mapping (Gordon and Breach Science, Amsterdam, 2000).

3. Bunge, H. J. Texture Analysis in Materials Science-Mathematical Methods 2nd edn (Butterworths, London, 1982).

4. Nokinov, V. Grain Growth and Control of Microstructure and Texture in Polycrystalline Materials (CRC, Boca Raton, 1997).

5. Thompson, C. V. \& Carel, R. Texture development in polycrystalline thin films. Mater. Sci. Eng. B 32, 211-219 (1995).

6. Harper, J. M. E. \& Rodbell, K. P. Microstructure control in semiconductor metallization. J. Vac. Sci. Technol. B 15, 763-779 (1997).

7. Dong, L. \& Srolovitz, D. J. Texture development mechanisms in ion beam assisted deposition. J. Appl. Phys. 84, 5261-5269 (1998).

8. Murray, C. E. \& Rodbell, K. P. Texture inheritance in $\mathrm{Al}(\mathrm{Cu})$ interconnect materials. J. Appl. Phys. 89, $2337-2342(2001)$.

9. Lucadamo, G., Barmak, K. \& Rodbell, K. P. Texture in Ti/Al and Nb/Al multilayer thin films: role of Cu. J. Mater. Res. 16, 1449-1459 (2001).

10. Schell, N., Matz, W., Bottiger, J., Chevallier, J. \& Kringhoj, P. Development of texture in TiN films by use of in situ synchrotron x-ray scattering. J. Appl. Phys. 91, 2037-2044 (2002).

11. Bulle-Lieuwma, C. W. T., van Ommen, A. H., Hornstra, J. \& Aussems, C. N. A. M. Observation and analysis of epitaxial growth of $\mathrm{CoSi}_{2}$ on (100) Si. J. Appl. Phys. 71, 2211-2224 (1992).

12. Bulle-Lieuwma, C. W. T. Epitaxial growth of $\mathrm{CoSi}_{2} / \mathrm{Si}$ structures. Appl. Surf. Sci. 68, 1-18 (1993).

13. Harper, J. M. E., Rodbell, K. P., Colgan, E. G. \& Hammond, R. H. Control of in plane texture of body centered cubic metal thin films. J. Appl. Phys. 82, 4319-4326 (1997).

14. Dong, L., Srolovitz, D. J., Was, G. S., Zhao, Q. \& Rollett, A. D. Combined out of plane and in plane texture control in thin films using ion beam assisted deposition. J. Mater. Res. 16, 210-216 (2001). 
15. Özcan, A. S. et al. Texture of TiSi 2 thin films on Si(001). J. Appl. Phys. 92, 5011-5018 (2002).

16. Tsukada, M. \& Ohfuji, S. Structural inheritance from polycrystalline underlayers in the growth of double-layered aluminum films. J. Vac. Sci. Technol. B 11, 326-332 (1993).

17. Tracy, D. P., Knorr, D. B. \& Rodbell, K. P. Texture in multilayer metallization structures. J. Appl. Phys. 76, 2671-2680 (1994)

18. Knorr, D. B., Merchant, S. M. \& Biberger, M. A. Development of texture in interconnect thin film stacks. J. Vac. Sci. Technol. B 16, 2734-2744 (1998).

19. Joint Committee on Powder Diffraction Standards. Powder Diffraction File 38-0844 (International Centre for Diffraction Data, Philadelphia, 1998).

20. Kilaas, R. Optimal and near-optimal filter in high-resolution electron microscopy. J. Microsc. 190, 45-51 (1998).

Supplementary Information accompanies the paper on www.nature.com/nature.

Acknowledgements We thank F. M. d'Heurle, J. M. E. Harper, C. Cabral, K. Ludwig, C. Murray, C. Noyan and K. P. Rodbell for discussions, and C. Diareo for TEM sample preparation. C.D. thanks the 'Fonds voor Wetenschappelijk Onderzoek-Vlaanderen' for support. The work at NCEM was supported by the Director, Office of Science, Office of Basic Energy Sciences, Division of Materials Sciences and Engineering, of the US Department of Energy. Pole figures were measured at the NSLS, Brookhaven National Laboratory, which is supported by the US Department of Energy, Division of Materials Sciences and Division of Chemical Sciences.

Competing interests statement The authors declare that they have no competing financial interests

Correspondence and requests for materials should be addressed to C.D.

(christophe.detavernier@ugent.be) or C.L. (clavoie@us.ibm.com).

\section{Coupled spatial variations in precipitation and long-term erosion rates across the Washington Cascades}

\section{Peter W. Reiners ${ }^{1}$, Todd A. Ehlers ${ }^{2}$, Sara G. Mitchell ${ }^{3}$ \& David R. Montgomery}

${ }^{1}$ Department of Geology and Geophysics, Yale University, 210 Whitney Avenue, New Haven, Connecticut 06511, USA

${ }^{2}$ Department of Geological Sciences, University of Michigan, Ann Arbor, Michigan 48109, USA

${ }^{3}$ Department of Earth and Space Sciences, University of Washington, Seattle, Washington 98195, USA

Past studies of tectonically active mountain ranges have suggested strong coupling and feedbacks between climate, tectonics and topography ${ }^{1-5}$. For example, rock uplift generates topographic relief, thereby enhancing precipitation, which focuses erosion and in turn influences rates and spatial patterns of further rock uplift. Although theoretical links between climate, erosion and uplift have received much attention ${ }^{2,6-10}$, few studies have shown convincing correlations between observable indices of these processes on mountain-range scales ${ }^{11,12}$. Here we show that strongly varying long-term $\left(>10^{6}-10^{7} \mathrm{yr}\right)$ erosion rates inferred from apatite $(\mathrm{U}-\mathrm{Th}) / \mathrm{He}$ cooling ages across the Cascades mountains of Washington state closely track modern mean annual precipitation rates. Erosion and precipitation rates vary over an order of magnitude across the range with maxima of $0.33 \mathrm{~mm} \mathrm{yr}^{-1}$ and $3.5 \mathrm{myr}^{-1}$, respectively, with both maxima located $50 \mathrm{~km}$ west (windward) of the topographic crest of the range. These data demonstrate a strong coupling between precipitation and long-term erosion rates on the mountain-range scale. If the range is currently in topographic steady state, rock uplift on the west flank is three to ten times faster than elsewhere in the range, possibly in response to climatically focused erosion.

The Washington Cascades provide a natural laboratory for observing interactions between climate, tectonics and topography. Although isolated volcanoes in the mountain range are part of an active magmatic arc extending south into California, most of the topographic relief in the Washington Cascades, and essentially all of it north of about $47^{\circ} \mathrm{N}$, is the result of deep-seated bedrock uplift and the resultant erosion, rather than volcanic extrusion. Summits in the range seldom reach elevations higher than $2.7 \mathrm{~km}$, but local relief is commonly $1.2-1.8 \mathrm{~km}$. The modern Washington Cascades cast a dramatic orographic rain shadow. Mean annual precipitation rates on the windward west flank are as high as $4 \mathrm{~m} \mathrm{yr}^{-1}$ and host lush vegetation, in contrast to rates of $0.2 \mathrm{~m} \mathrm{yr}^{-1}$ or less in the sagebrush steppe directly east of the range. Palaeobotanical evidence $^{13}$ indicates that the orographic rain shadow was not as strong before the Late Miocene epoch, so it is likely that at least some of the current topographic expression of the Washington Cascades is younger than 10-15 Myr. This is consistent with the well-known post-15-Myr BP warping and uplift of the lava flows of the eastderived Columbia River basalt group on the eastern side of the range. Little is known about either the kinematics or dynamics of uplift in the Washington Cascades. Global positioning system (GPS) data suggest only weak horizontal crustal shortening across the range $^{14}$, and patterns of crustal seismicity and detailed geologic mapping do not suggest any large active Neogene faults or other structures (see the catalogued seismicity record at $\langle$ http://www.seismo.berkeley.edu/seismo/ $>$; also available in the Supplementary Information $)^{15-19}$.

We measured bedrock apatite (U-Th)/He ages from samples in a broad $(200 \mathrm{~km})$ east-west swath across the Washington Cascades to examine spatial patterns of erosion in a setting with a strong orographic precipitation gradient. The apatite (U-Th)/He system has a closure temperature of $\sim 60-70^{\circ} \mathrm{C}$ (ref. 20) and ages generally represent the time since a rock passed through a depth in the crust corresponding to that temperature (typically $1.5-2.5 \mathrm{~km}$ ), as a result of tectonic or erosional exhumation.

Thirty new apatite He ages from twenty samples from the western flank of the Cascades are shown in Fig. 1, along with our existing data $^{21}$ from elsewhere in this swath. Samples were collected from plutons with crystallization ages ranging from $\sim 20-95 \mathrm{Myr}$ BP. In all cases except that of Mt Pilchuck in the far west, apatite He ages are much less than crystallization ages. In this part of the Cascades, plutons younger than $\sim 20 \mathrm{Myr} \mathrm{BP}$ are small and rare and there is no evidence in age and pluton distributions that ages reflect a significant component of magmatically controlled heating.

Apatite He ages in this swath of the central Cascades vary from 4.4 to $60 \mathrm{Myr}$ в (Fig. 1). In single vertical transects collected over short horizontal distances, ages generally show increasing ages, or little change in age, with increasing elevation ${ }^{21}$. The most obvious pattern in the data consists of relatively old ages ( $>25 \mathrm{Myr} \mathrm{BP})$ at the topographic crest and far eastern and western flanks of the range, and young ages ( $<12 \mathrm{Myr} \mathrm{BP})$ on the west slope (Figs 1 and 2$)$.

To first order, the ratio of the apatite He closure isotherm depth $(\sim 1.5-2.5 \mathrm{~km})$ and age yields an estimate of time-averaged exhumation rate. As there are no known late Tertiary faults or extensional structures in this region ${ }^{15-19}$, we assume that this exhumation is entirely erosional. We calculated erosion rates for these samples by relating each apatite $\mathrm{He}$ age to cooling-ratedependent closure temperature and depth through a series of equations with assumed parameters, including geothermal gradient, He diffusion properties ${ }^{20}$, thermal diffusivity, and depth to constant temperature ${ }^{22}$ (see Supplementary Information). We used sample-specific geothermal gradients based on interpolation of observed gradients ranging from 20 to $40^{\circ} \mathrm{C} \mathrm{km}^{-1}$ (ref. 23) (Fig. 1). Our model assumes a steady-state distribution of isotherms in the crust, which is fairly robust for the relatively slow erosion rates inferred from these data and tectonic setting over the past $40 \mathrm{Myr}$ (ref. 23).

Closure depth for each sample was adjusted to account for local 